Preprint IASSNS-HEP-98/001

\title{
Cyclotron resonance in a two-dimensional electron gas with long-range randomness
}

\author{
M. M. Fogler \\ School of Natural Sciences, Institute for Advanced Study, Olden Lane, Princeton, NJ 08540 \\ B. I. Shklovskii \\ Theoretical Physics Institute, University of Minnesota, 116 Church St. Southeast, Minneapolis, Minnesota 55455
}

(June 18, 2018)

\begin{abstract}
We show that the the cyclotron resonance in a two-dimensional electron gas has non-trivial properties if the correlation length of the disorder is larger than the de Broglie wavelength: (a) the lineshape assumes three different forms in strong, intermediate, and weak magnetic fields (b) at the transition from the intermediate to the weak fields the linewidth suddenly collapses due to an explosive growth in the fraction of electrons with a diffusive-type dynamics.
\end{abstract}

The cyclotron resonance (CR) is one of the basic tools for studying the electronic properties of physical systems in an external magnetic field. A very interesting example of such a system is a two-dimensional electron gas (2DEG), which exhibits the quantized Hall effect if the magnetic field is sufficiently strong. The CR can be studied by measuring the transmission of an electromagnetic signal of some frequency $\omega$ through the 2DEG. If the absorption is weak, the change in the transmission is proportional to the real part of the dynamical conductivity $\sigma_{x x}(\omega)$, which is the average over the active and inactive circular polarizations, $\operatorname{Re} \sigma_{x x}(\omega)=\left[\operatorname{Re} \sigma_{+}(\omega)+\right.$ $\left.\operatorname{Re} \sigma_{-}(\omega)\right] / 2$ (for linearly polarized signals). The active polarization's contribution $\operatorname{Re} \sigma_{+}(\omega)$ has a peak at $\omega$ close to the cyclotron frequency $\omega_{c}$ of the external magnetic field. The disorder-related zero-temperature width and shape of this peak are the subjects of this Letter.

The theoretical study of the CR in the 2DEG has been initiated by Ando [1]. Although many pieces of the complete picture has been put in place by him and later by other authors [2,3], the consistent description of this phenomenon exists only for the case where the correlation length $d$ of the random potential $U(x, y)$ acting on the electrons in the disordered 2DEG is smaller than the de Broglie wavelength $k_{F}^{-1}\left(k_{F}\right.$ is the Fermi wavevector in zero magnetic field). In this case the effect of the random potential is described by a single quantity, the transport (or momentum relaxation) time $\tau$. It was understood, however, that the conventional Drude-Lorentz formula

$$
\operatorname{Re} \sigma_{+}(\omega)=\frac{\sigma_{0}}{1+\left(\omega-\omega_{c}\right)^{2} \tau^{2}}
$$

( $\sigma_{0}$ is the zero-field conductivity), which gives the Lorentzian peak with the half width at half maximum (HWHM) of $\tau^{-1}$, applies only in the uninteresting case $\omega_{c} \tau \ll 1$. In the other limit $\left(\omega_{c} \tau \gg 1\right)$ the CR lineshape is non-Lorentzian and has a much larger width [1]

$$
\Delta \omega_{1 / 2}=0.73 \sqrt{\omega_{c} / \tau}
$$

due to the formation of discrete Landau levels. This behavior of $\Delta \omega_{1 / 2}$ is illustrated by the thin line in Fig. 1.

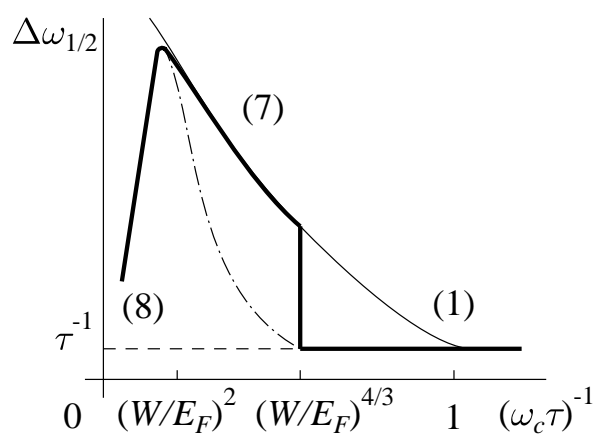

FIG. 1. Dependence of the CR linewidth on $\left(\omega_{c} \tau\right)^{-1}$, the quantity inversely proportional to the magnetic field. Thick line: our results for the long-range potential with given $W / E_{F}$ and $\tau$ [for the case $k_{F} d \gg\left(E_{F} / W\right)^{2 / 3}$ ]. Labels (1), ( ( $)$, and ( 8 ) correspond to the equation numbers. Thin line: short-range potential with the same $\tau$. Dash-dotted line: the width $\nu$ of an additional structure near the very resonance (see text).

Here and below $\Delta \omega_{1 / 2}$ is the median width defined by

$$
\int_{0}^{\Delta \omega_{1 / 2}} d \omega \operatorname{Re} \sigma_{+}\left(\omega+\omega_{c}\right)=\frac{1}{2} \int_{0}^{\infty} d \omega \operatorname{Re} \sigma_{+}\left(\omega+\omega_{c}\right)
$$

Using the median width instead of the conventional HWHM is more adequate because the CR lineshape can be rather intricate for a long-range random potential.

For simplicity, we will consider a model of a Gaussian random potential whose correlator decays sufficiently fast at $r>d$ and does not possess any other characteristic scales besides $d$. The rms amplitude of $U$ will be denoted by $W$. Until the very end we will assume that $k_{F} d \gg\left(E_{F} / W\right)^{2 / 3}$, where $E_{F}$ is the Fermi energy. As one can see from Fig. 1 illustrating our results for this case, the dependence of $\Delta \omega_{1 / 2}$ on the magnetic field is non-monotonic. Even more remarkable, $\Delta \omega_{1 / 2}$ exhibits 
a rapid collapse to its classical value of $\tau^{-1}$ in the vicinity of the point $\omega_{c} \tau \sim\left(E_{F} / W\right)^{2 / 3} \gg 1$. The derivation of these results is based on the picture of the "classical localization" [何, which we will now discuss.

If the random potential $U$ is smooth and the time scale on which we study the motion of an electron is not too long, then it can be described classically, as a motion of a single particle with energy $E_{F}$. (We neglect the interaction). If the magnetic field is not too low, the motion can be decomposed into a fast cyclotron gyration and a slow drift of the guiding center $\boldsymbol{\rho}=\left(\rho_{x}, \rho_{y}\right)$ of the cyclotron orbit. For magnetic field in the negative $z$-direction, $\rho_{x}=x+\left(v_{y} / \omega_{c}\right)$ and $\rho_{y}=y-\left(v_{x} / \omega_{c}\right)$, where $x$ and $y$ are the coordinates of the electron and $\boldsymbol{v}=-v_{F}(\sin \theta, \cos \theta)$ is its velocity. We will call the magnetic field strong if the cyclotron radius $R_{c}=v_{F} / \omega_{c}$ is smaller than $d$. It is usually assumed that in such strong fields the guiding center drifts along a level line $U(\boldsymbol{\rho})=$ const of the random potential, which is typically a closed loop of size $d$. Recently it has been realized [4] 5] that the drift approximation is also valid in the intermediate field regime $1<R_{c} / d<\left(E_{F} / W\right)^{2 / 3}$ [the same as $\left(E_{F} / W\right)^{4 / 3}<\omega_{c} \tau<\left(E_{F} / W\right)^{2}$ because $\left.\tau \sim\left(d / v_{F}\right)\left(E_{F} / W\right)^{2}\right]$. The point is that in this regime the cyclotron gyration is still sufficiently fast, so that the guiding center remains practically "frozen" during one cyclotron period. Therefore, the guiding center motion is determined by $U_{0}$, the random potential averaged over the cyclotron orbit,

$$
U_{0}\left(\boldsymbol{\rho}, R_{c}\right)=\int_{0}^{2 \pi} \frac{d \theta}{2 \pi} U\left(\rho_{x}+R_{c} \cos \theta, \rho_{y}+R_{c} \sin \theta\right) .
$$

The guiding center is still bound to one of the level lines, but those are the level lines of $U_{0}$, not $U$ 酒. The rms amplitude $W_{0}$ of the potential $U_{0}$ is by a factor of $\sqrt{d / R_{c}}$ smaller than $W$ due to the averaging, but the correlation length is the same; therefore, a typical level line of $U_{0}$ is still a loop of size $d$. The frequency $\omega_{d}$ of the guiding center motion along such a loop (the drift frequency) is given by $W_{0} /\left(m \omega_{c} d^{2}\right)$, which can be cast into the form

$$
\omega_{d}=\left(R_{c} / d\right) \sqrt{\omega_{c} / \tau}, \quad R_{c} \gg d .
$$

Obviously, the electrons on the periodic orbits are (classically) localized and do not participate in the DC transport. However, a more accurate analysis [4] reveals that a very small fraction of the order of $e^{-\left(\omega_{c} / \omega_{d}\right)^{2 / 3}}$ of the trajectories remains delocalized. Such trajectories form a stochastic web in the vicinity of the percolation contour. The stochastic web rapidly grows with decreasing magnetic field and turns into a stochastic sea at

$$
R_{c} / d=\left(E_{F} / W\right)^{2 / 3},
$$

where $\omega_{d}=\omega_{c}$. In even lower magnetic fields (the weak field regime) the stochastic sea spans almost the entire phase space. Correspondingly, the static conductivity is exponentially small, $\sigma_{x x}(0) \propto e^{-\left(\omega_{c} / \omega_{d}\right)^{2 / 3}}$ when $\omega_{d} \ll \omega_{c}$ (the strong and the intermediate field regimes), rapidly blows up near $\omega_{d}=\omega_{c}$ point (the boundary of the intermediate and the weak field regimes), and finally crosses over to the Drude-Lorentz formula (11) in weak fields [6].

Our goal is to show that the dynamical conductivity also exhibits a rapid change near the $\omega_{d}=\omega_{c}$ point: the aforementioned collapse of the CR linewidth. This can be done on the basis of the classical formula for $\sigma_{+}(\omega)$,

$$
\sigma_{+}(\omega)=\frac{\sigma_{0}}{\tau} \int_{0}^{\infty} d t\left\langle e^{i \omega t-i[\theta(t)-i \theta(0)]}\right\rangle .
$$

Suppose that $\Delta \omega \equiv \omega-\omega_{c}$ is smaller than $\omega_{c}$ by absolute value, then the full equation of motion for $\theta$ can be replaced by its average over the cyclotron period,

$$
\frac{d \theta}{d t}=\omega_{c}+\delta \omega, \quad \delta \omega=\left.\frac{1}{m \omega_{c} R} \frac{\partial}{\partial R} U_{0}(\boldsymbol{\rho}, R)\right|_{R=R_{c}}
$$

where $\delta \omega$ is the local correction to the gyration frequency of the velocity vector. The origin of such a correction is quite clear. Indeed, the random potential creates an additional centripetal force, which is either parallel to the Lorentz force and thus speeds up the cyclotron gyration, or anti-parallel, which slows it down.

In the strong and intermediate field regimes most of the guiding centers are localized on the periodic orbits of small size $\lesssim d$. The correction $\delta \omega$ does not vary much on this length scale. In fact, to find $\Delta \omega_{1 / 2}$ up to a numerical factor, we can use the approximation $\delta \omega=$ const. Substituting this into Eqs. (5) and (6), we find that $\operatorname{Re} \sigma_{+}(\omega)$ has the form of a Gaussian peak of width $[\delta \omega]_{\mathrm{rms}}$, and so

$$
\begin{aligned}
\Delta \omega_{1 / 2} \sim[\delta \omega]_{\mathrm{rms}} & =\operatorname{const} \frac{W}{m \omega_{c} d^{2}}, \quad R_{c} \ll d, \\
& =\sqrt{\omega_{c} /(\pi \tau)}, \quad R_{c} \gg d .
\end{aligned}
$$

The broadening of the $\mathrm{CR}$ line is of the inhomogeneous type, and comes from the places where $\Delta \omega=\delta \omega$ [ Equations (7) and (8) show that $\Delta \omega_{1 / 2}$ linearly increases as a function of $\left(\omega_{c} \tau\right)^{-1}$ in the strong-field regime, reaches its maximum at $R_{c} \sim d$, and then decreases in

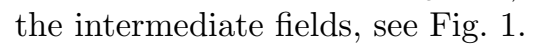

The situation is different in the weak fields where most of the trajectories are extended and ergodic. In this case the broadening is of the homogeneous type and is much smaller than $[\delta \omega]_{\mathrm{rms}}$ because of the motional narrowing. The crossover between the two types of broadening occurs at the low-field end of the intermediate-field regime, which we study below. (However, a broader physical discussion will be resumed in the concluding remarks).

According to Eqs. (3) and (8), $[\delta \omega]_{\mathrm{rms}} \ll \omega_{d}$ in this regime. Because of this inequality, the orbits of low frequency $\Omega \ll \omega_{d}$ whose perimeter length is typically 
$\left(\omega_{d} / \Omega\right) d$ turn out to be important. To show this we present $\sigma_{+}(\omega)$ as follows,

$$
\begin{aligned}
\sigma_{+}(\omega) & =\frac{\sigma_{0}}{\tau} \int_{0}^{\infty} d \Omega f(\Omega) I(\Omega, \Delta \omega), \\
I(\Omega, \Delta \omega) & =\int_{0}^{\infty} d t\left\langle\exp \left[i \Delta \omega t-i \int_{0}^{t} d t^{\prime} \delta \omega\left(t^{\prime}\right)\right]\right\rangle_{\Omega},
\end{aligned}
$$

where $\langle\ldots\rangle_{\Omega}$ denotes the average over the orbits of frequency $\Omega$, and $f(\Omega)$ is the probability density of finding an electron with energy $E_{F}$ on such an orbit (with the convention $\Omega=+0$ for the unbound trajectories of the stochastic web). The correlations in $\delta \omega(\boldsymbol{\rho})$ decay sufficiently fast $\left(\sim \rho^{-3}\right)$ with distance; hence, the high-order correlators of $\delta \omega$ can be neglected, and we arrive at

$$
I \simeq \int_{0}^{\infty} d t \exp \left[i \Delta \omega t-\frac{1}{2} \int_{0}^{t} d t_{1} \int_{0}^{t} d t_{2}\left\langle\delta \omega\left(t_{1}\right) \delta \omega\left(t_{2}\right)\right\rangle_{\Omega}\right] .
$$

For the same reason we can approximate the second-order correlator by the sum of isolated short pulses,

$$
\left\langle\delta \omega\left(t_{1}\right) \delta \omega\left(t_{2}\right)\right\rangle_{\Omega}=\sum_{n=-\infty}^{\infty} P\left(t_{1}-t_{2}-\frac{2 \pi n}{\Omega}\right),
$$

where $P(0)=\left\langle\delta \omega^{2}\right\rangle$ and $P(t) \ll P(0)$ for $|t| \gg \omega_{d}^{-1}$. In fact, if both $\Omega$ and $|\Delta \omega|$ are much smaller than $\omega_{d}$, the actual functional form of the pulses is unimportant, and we can approximate $P(t)$ by $2 \nu \delta(t)$, where

$$
\nu \sim\left\langle\delta \omega^{2}\right\rangle / \omega_{d} \sim \omega_{c} /\left(\omega_{d} \tau\right)
$$

After such approximations and some algebra we find:

$$
\begin{aligned}
\operatorname{Re} I & =\frac{\pi}{\sqrt{2 \Omega \nu}} \sum_{n=-\infty}^{\infty} c_{n} \exp \left[-\frac{\pi(\Delta \omega-n \Omega)^{2}}{2 \Omega \nu}\right], \\
c_{n} & =\int_{0}^{1} d x \cos (\pi n x) \exp \left[-\frac{\pi \nu\left(2 x-x^{2}\right)}{2 \Omega}\right] .
\end{aligned}
$$

If $\Omega \gg \nu$, then $c_{0} \simeq 1$ and $c_{n} \simeq \nu /\left(\pi n^{2} \Omega\right), n \neq 0$, so that $\operatorname{Re} I(\Omega, \Delta \omega)$ as a function of $\Delta \omega$ is the sum of narrow Gaussians centered at equidistant points $\Delta \omega=n \Omega$. In the opposite limit the Gaussians merge into a single Lorentzian,

$$
\operatorname{Re} I(\Omega, \Delta \omega)=\frac{\nu}{(\Delta \omega)^{2}+\nu^{2}}, \quad \Omega \ll \nu,|\Delta \omega| \ll \omega_{d} .
$$

There is a simple way to understand this equation. As discussed above, the random potential modifies the local gyration frequency of the velocity vector by $\delta \omega$. The sign of the correction changes randomly each time the guiding center travels the distance $\sim d$. This causes the angular diffusion of the velocity vector in addition to the regular precession with frequency $\omega_{c}$ and therefore, the momentum relaxation. The time $\omega_{d} /\left\langle\delta \omega^{2}\right\rangle=\nu^{-1}$ plays the role of the effective transport time, and so Eq. (15) is expected by analogy with Eq. (11).

To finish the calculation of $\sigma_{+}(\omega)$ we need to know $f(\Omega)$. It is determined by the statistical properties of the potential $U$, viz., by the exponent $H$ in the equation

$$
\int_{q}^{2 q} d q q J_{0}^{2}\left(q R_{c}\right) \tilde{C}(q) \propto q^{-2 H}
$$

where $J_{0}$ is the Bessel function and $\tilde{C}(q)$ is the Fourier transform of $C(\boldsymbol{r}) \equiv\langle U(0) U(\boldsymbol{r})\rangle$. In a practically relevant case where the potential is created by randomly positioned donors confined to a narrow layer separated by the distance $d$ from the 2DEG, $\tilde{C}(q)=\tilde{C}(0) e^{-2 q d}$, so that $H=-1$ if $q \ll R_{c}^{-1}$ and $H=-1 / 2$ if $R_{c}^{-1} \lesssim q \lesssim d^{-1}$. In both cases

$$
\begin{aligned}
f(\Omega) & \simeq s \Omega^{-1}\left(\Omega / \omega_{d}\right)^{s}, \quad \Omega_{\mathrm{web}}<\Omega<\omega_{d} \\
& \simeq\left(\Omega_{\mathrm{web}} / \omega_{d}\right)^{s} \delta(\Omega-0) \quad \text { at other } \Omega
\end{aligned}
$$

where $s$ is very close to $1 / 2$ [see Isichenko [8] for details; $\Omega f(\Omega)$ corresponds to his $F(\Omega)$ ]. The quantity $\Omega_{\mathrm{web}} \sim \omega_{d} e^{-\left(\omega_{c} / \omega_{d}\right)^{2 / 3}}$ is the drift frequency of such orbits within the stochastic web that give the dominant contribution [4] to the DC transport.

We can now substitute Eqs. (13 16 ) into Eq. (9). Since $s<1$, only the $n=0$ term in Eq. (13) is important for $\nu \lesssim \Omega \lesssim \omega_{d}$. Also, it is safe to assume that $\omega_{d} / \nu \ll e^{2 /(2 s-1)}[9]$. With this in mind, we obtain $\operatorname{Re} \sigma_{+}(\omega)=\left(\sigma_{0} / \nu \tau\right)\left[S_{1}(\omega)+S_{2}(\omega)\right]$, where

$$
\begin{aligned}
& S_{1}(\omega) \approx \sqrt{\frac{\Omega_{c}}{\omega_{d}}} \frac{1}{1+(\Delta \omega / \nu)^{2}},|\Delta \omega| \lesssim \omega_{d}, \\
& S_{2}(\omega) \approx \sqrt{\frac{\pi^{2} \nu}{8 \omega_{d}}} \ln \left[\frac{\omega_{d} \nu}{\max \left\{(\Delta \omega)^{2}, \Omega_{c} \nu\right\}}\right],|\Delta \omega| \lesssim \sqrt{\omega_{d} \nu},
\end{aligned}
$$

$\Omega_{c}=\max \left\{\Omega_{\mathrm{web}}, \nu\right\}$. It is easy to see that as long as $\Omega_{\mathrm{web}} \leq \nu \ll \omega_{d}, \Delta \omega_{1 / 2}$ is determined by $S_{2}(\omega)$, and thus is of the order of $\sqrt{\omega_{d} \nu} \sim \sqrt{\omega_{c} / \tau}$, in accordance with Eq. (8). The CR lineshape for this case is depicted in Fig. 2a.

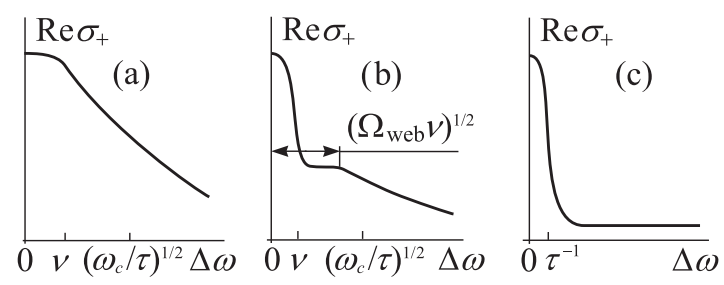

FIG. 2. The CR lineshape (schematically). (a) before the collapse (b) in the course of the collapse (c) immediately after the collapse. 
Let us now discuss the aforementioned collapse of the CR linewidth. $\Omega_{\text {web }}$ grows exponentially as the magnetic field decreases and eventually becomes larger than $\nu$. From this moment, the maximum value of $S_{2}$ rapidly decreases and that of $S_{1}$ does the opposite. It gives rise to a narrow Lorentzian peak at the center of the CR line (see Fig. 2b). At the $\omega_{d}=\omega_{c}$ point where $\Omega_{\mathrm{web}} \sim \omega_{d}$, it is this peak that determines $\Delta \omega_{1 / 2}$. Thus, $\Delta \omega_{1 / 2}$ drops to the value of $\nu=\omega_{c} /\left(\omega_{d} \tau\right)=\tau^{-1}$ (Fig. 2c). From this point on, i.e., in the weak-field regime, Eq. (11) applies.

Several comments are in order here. Formula (7) for the strong-field regime is not new (see Refs. [1 3]). However, its derivation has remained unsatisfactory: Ando's work [1] is based on the self-consistent Born approximation (SCBA), which is invalid in this regime; Prasad and Fujita [2] ignored the localized nature of the particle motion; Bychkov and Iordanskii [3] de facto assumed that the electron's trajectory (in the absence of interactions) is $\delta \omega(\boldsymbol{\rho})=$ const instead of the correct $U_{0}(\boldsymbol{\rho})=$ const.

So far we have studied the case $k_{F} d \gg\left(E_{F} / W\right)^{2 / 3}$. In the opposite limit an additional "quantum" region appears on the phase diagram of Fig. 3 [10]. Its boundary is formed by the lines $l=d$ where $l=\sqrt{\hbar / m \omega_{c}}$ is the magnetic length, and $\omega_{c} \tau_{q}=1$ where $\tau_{q} \sim \tau /\left(k_{F} d\right)^{2}$ is the quantum lifetime. The lower edge of this region, i.e., the line $k_{F} d=1$ was discussed in the beginning of this Letter. Now we will show that the collapse of the CR linewidth occurs in the case $1 \ll k_{F} d \ll\left(E_{F} / W\right)^{2 / 3}$ as well, and that its position on the phase diagram is given by the line $l=d$ (which is the same as $R_{c} / d=k_{F} d$ ). Contrary to the "classical" case, the quantum collapse takes place when the Landau levels are well separated.

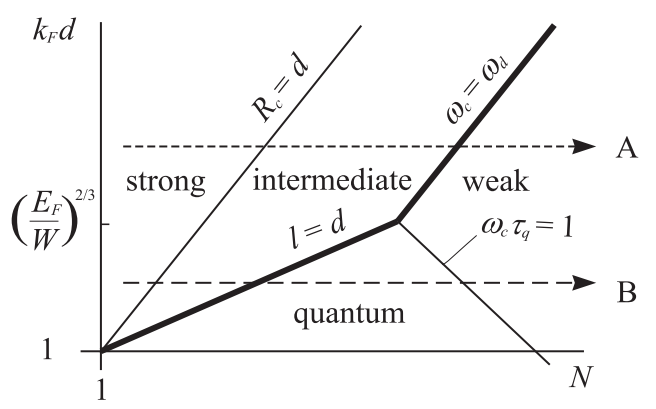

FIG. 3. Phase diagram of the CR (double log-scale). $N$ is the index of the Landau level closest to $E_{F}$. The bold line marks the location of the linewidth collapse. The paths $\mathrm{A}$ and $\mathrm{B}$, which the $2 \mathrm{DEG}$ follows as the magnetic field decreases, correspond to the classical (Fig. 1) and the quantum (Fig. 4) cases, respectively.

The CR lineshape in the quantum region can be studied within the SCBA. Doing the integration in Eq. (2.12) of Ref. [1] for the case of $E_{F}$ positioned between the Landau levels and $|\Delta \omega| \ll \sqrt{\omega_{c} / \tau_{q}}$, we find that the CR line is roughly Lorentzian, while its median width is given by

$$
\Delta \omega_{1 / 2}=0.66 \tau^{-1} \sqrt{\omega_{c} \tau_{q}}
$$

On the other hand, in the intermediate-field region $\Delta \omega_{1 / 2}$ is given by Eq. (8). Therefore, the crossing of the $l=d$ line causes the drop of $\Delta \omega_{1 / 2}$ by a large factor of $k_{F} d$ (Fig. 1). The physics of this "quantum" collapse is quite similar to that of the classical one: the crossing of the line $l=d$ is accompanied by an explosive growth of the quantum localization length of the states near the Landau level center (see [10] for discussion). The states with sufficiently large localization length contribute to $\sigma_{+}(\omega)$ in the form similar to Eq. (1) but with $\tau$ replaced by the effective transport time. Since the density of states at the Landau level center is larger than the zero-field density of states by a factor of $\sqrt{\omega_{c} \tau_{q}}$ [1], this effective transport time is smaller than $\tau$ by the same factor in agreement with Eq. (17).

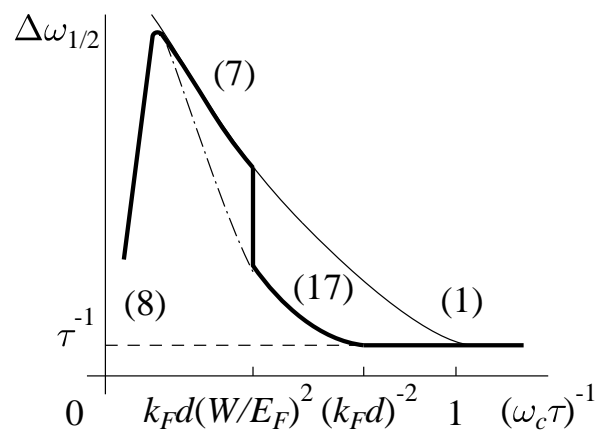

FIG. 4. Same as Fig. 目 but for the path B of Fig. B.

Until now we considered $W$ a fixed parameter of the theory. In a more realistic model (see above) $W$ is determined by the concentration $n_{i}$ of randomly positioned donors and the screening properties of the 2DEG. Away from the strong-field regime, the screening is very much the same as in zero field, and one obtains $W \sim E_{F} \sqrt{n_{i}} /\left(k_{F}^{2} d\right)$. Thus, the "quantum" case $k_{F} d \ll$ $\left(E_{F} / W\right)^{2 / 3}$ is realized if $n_{i}$ is sufficiently low, $n_{i} \ll k_{F} / d$. On the other hand, in the strong-field regime (far from the collapse), the amount of screening, $W$, and $\Delta \omega_{1 / 2}$ oscillate with the filling factor [1]. A consistent description of such oscillations must take into account the nonlinear screening effects 12].

In conclusion, let us discuss the experimental side. The non-monotonic dependence of $\Delta \omega_{1 / 2}$ on the magnetic field with the maximum at $R_{c} \sim d$ has been observed in Ref. [13], in agreement with our theory. Arguably, the collapse of the CR linewidth has also been seen (for the $400 \AA$ spacer sample). However, a decisive confirmation of the latter prediction requires further experiments.

M. M. F. is supported by DOE Grant No. DE-FG0290ER40542 and B. I. S. by NSF Grant No. DMR9616880. We thank A. Yu. Dobin, M. I. Dyakonov, Yu. M. Galperin, and A. A. Koulakov for very useful discussions. 
[1] T. Ando, J. Phys. Soc. Jpn. 38, 989 (1975).

[2] M. Prasad and S. Fujita, Physica 91A, 1 (1978).

[3] Yu. A. Bychkov and S. V. Iordanskii, Fiz. Tverd. Tela 30, 794 (1988) [Sov. Phys. Solid State 30, 455 (1988)].

[4] M. M. Fogler, A. Yu. Dobin, V. I. Perel, and B. I. Shklovskii, Phys. Rev. B 56, 6823 (1997).

[5] B. Laikhtman, Phys. Rev. Lett. 72, 1060 (1994).

[6] We are talking about $\sigma_{x x}(0)$ on not too large length scales where the quantum localization effects can be neglected.

[7] A more careful approach confirms that $\Delta \omega_{1 / 2} \sim[\delta \omega]_{\mathrm{rms}}$ but refutes the Gaussian lineshape as an artifact of the $\delta \omega=$ const approximation.

[8] M. B. Isichenko, Rev. Mod. Phys. 64, 961 (1992).

[9] For $H=-1 / 2$ the theory of Ref. 8 gives $s=4(1-$ $H) /(10-3 H)=12 / 23$, so that $e^{2 /(2 s-1)} \approx 10^{20}$.

[10] M. M. Fogler, A. Yu. Dobin, and B. I. Shklovskii, Phys. Rev. B 57, xxxx (1998).

[11] See, e.g., T. Ando and Y. Murayama, J. Phys. Soc. Jpn. 54, 1519 (1985); D. Heitmann, M. Zeismann, and L. L. Chang, Phys. Rev. B 34, 7463 (1986).

[12] M. M. Fogler and B. I. Shklovskii, Phys. Rev. B 52, 17366 (1995), Sec. III.

[13] M. Watts, I. Auer, R. J. Nicholas, J. J. Harris, and C. T. Foxon, in High Magnetic Fields in Semiconductor Physics III (Springer-Verlag, Berlin, 1992) edited by G. Landwehr, p. 581. 